\title{
ARIS: A computer-assisted instruction system
}

\author{
M. PAVEL, S. MARCOVICI, A. SHERMAN, and J.-C. FALMAGNE \\ New York University, New York, New York 10003
}

\begin{abstract}
The automated real-time instructional system (ARIS) is a small computer-assisted instructional system written to run under the UNIX operating system on a PDP-11/34A machine. ARIS is a tool used to provide highly individualized instruction and, at the same time, to study the process of learning by keeping detailed records of students' actions. Currently, the system is used to teach logic and set theory. This paper describes the general philosophy, operation, and implementation of ARIS.
\end{abstract}

Teaching quantitative skills to graduate students in psychology programs is often a very difficult and thankless task. There are two major problems. The first is due to the wide range of backgrounds of students. Some of the students have not been exposed to any mathematics beyond their high school education; others are quite sophisticated. The second problem is related to the classical aversion of students toward mathematical knowledge; that is, some students are convinced that they cannot learn mathematics and are consequently quick to give up and let the class proceed without them.

The attempt to minimize the impact of these problems led us to consider a computer-based instruction system. Since at the time there were no systems satisfying our requirements, our group at New York University (NYU) began the development of a computerassisted instruction (CAI) system. The goals of the project closely paralleled the need to solve these two problems: (1) to individualize instruction and allow each individual to progress at his own pace, and (2) to develop a research tool to study and possibly optimize the process of learning.

The result of these efforts is ARIS (automated realtime instructional system). The currently operational version of ARIS consists of the basic system and courseware covering the first part of an introductory course in mathematics. In particular, the courseware includes (1) sentential logic, including axiomatic analyses with formal theorem proving, (2) quantifier logic, and (3) set theory.

To date, the system has been successfully used in three semesters of a graduate course and a summer course for high school students.

Although the original design aims were rather modest, ARIS has a number of features that compare favorably with much larger systems: (1) Adaptive material pre-

This work was supported in part by a grant from the EXXON foundation. The authors wish to acknowledge the work of Martin Gizzi and Charlie Chubb, who played a major part in debugging of the system and running the courses. We also thank Geoff Iverson and Mark Perkins, who spent many months participating in the development of the courseware.
sentation-The student's past performance is used to determine the next material to be presented. The speed of progress can be adjusted by both the student and ARIS. However, ARIS will not allow a student to proceed until a minimum criterion is reached. Thus, at any given time, students may be at very different places in the course, but they will not differ very much in the precision of their knowledge. (2) On-line proof checkerARIS has a special facility to teach one of the more difficult skills, formal theorem proving. As the student types in his proof line by line at a terminal, each line is parsed and checked for correctness. This approach assures that each step is correct and that no steps are skipped. (3) Homework-A significant portion of a student's work under ARIS is assigned as homework. The educational value of the work that a student does away from the machine may have been greatly underestimated in the development of other CAI systems. At home, with paper and pencil, the student can no longer rely on the immediate detection of each error. Instead, the student himself must search through many steps or operations to find his errors. The student is thereby encouraged to learn strategies for self-correction. (4) Economy and portability-ARIS is designed to run on a small machine or a larger microprocessor, and it is written in an efficient and portable language. In addition, the homework assignments that are done off the machine contribute to a higher efficiency of the system.

\section{LEARNING PROCESS OVERVIEW}

The interaction the student and ARIS is divided into three phases, depicted on Figure 1. The student loops through these phases sequentially as each topic is mastered: (1) Text presentation and on-line quizzingThis phase represents a typical CAI interaction. The student is presented with a section of course material and occasionally is asked to answer simple questions or to solve easy problems. If the student is doing well and feels that there is no need for the explanatory text, he can skip the review and proceed directly to a short quiz. The results of the quizzing are used to determine 


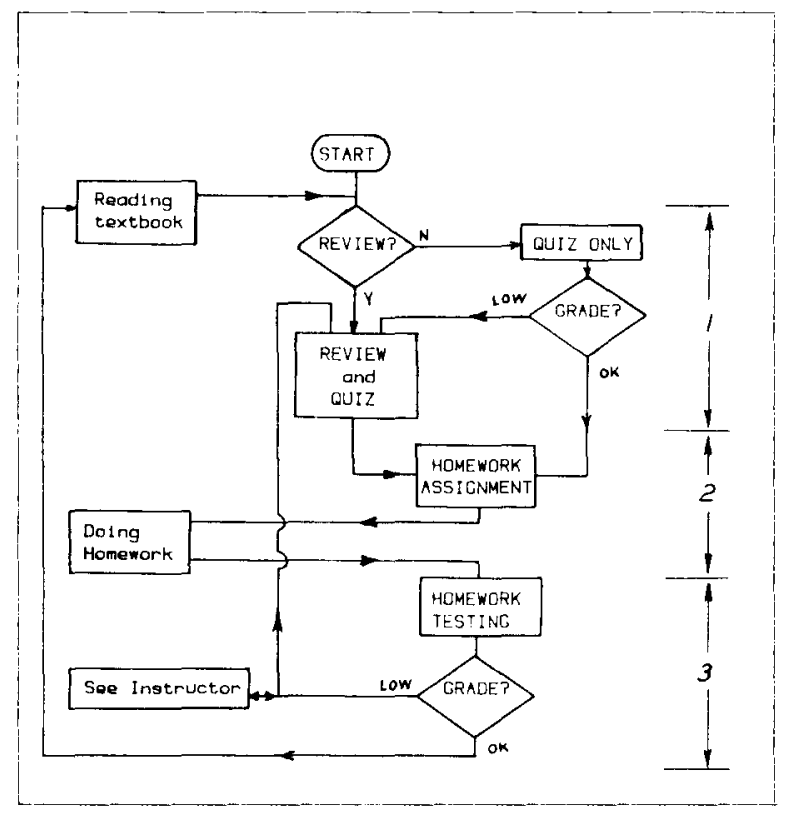

Figure 1. A simplified flow diagram of the interaction between a student and ARIS. The learning process consists of three phases indicated on the right side of the figure. The three boxes on the left side of the diagram correspond to the activities of the student away from the terminal.

the amount and the difficulty of the homework assigned in Phase 2. (2) Homework assignment-After the completion of the review and quiz, ARIS selects an appropriate number of homework problems and prints out the assignment. Each student receives an individualized assignment: Even the same type of problem assigned twice or to different students will require different answers. A more capable student would receive smaller, but more challenging homework assignments. The assignment consists of a general question to be answered by the student. (3) Homework checking and scoringWhen the student returns to the system, he will first be asked to solve the homework problems assigned at the end of the previous session. The problems will again be displayed on the terminal, but this time, each with a set of specific questions and, often, with multiplechoice answers. Only if the student solved the problem successfully at home will he be able to answer these questions; they are too complex to be answered without preparation. If ARIS recieves an incorrect or partially incorrect answer, it will guide the student through the solution, explaining each step and, possibly, asking some further questions. This procedure improves the chances that the student will find the particular step in the solution on which he made an error.

In the current version of ARIS, each student is continuously evaluated and his absolute score is displayed on his screen. In addition, the student can find his relative standing in the class. Upon request, the system will display a histogram representing the current standing of all the students in the class and a pointer indicating the individual's current position.

\section{IMPLEMENTATION-HARDWARE}

The hardware supporting ARIS consists of a PDP-11/34A CPU with $128 \mathrm{~K}$ words of memory, two 5-MB RL-02 disks, and a DZ-11 serial line multiplexer. The system is sufficiently compact to require only one of the disks; the other is used for software backup. A student's interaction with ARIS is mediated via CRT terminals, and the hard-copy homework assignments are provided by a LA-36 DECwriter.

Both the terminal and the hard-copy output are dumb terminals, except that both were modified to enable the presentation of a special character set to display mathematical symbols. The DECwriter was enhanced by a commercially available Graphics II board manufactured by the Seinear Corporation, and the CRT terminals were modified in our electronics shop. Such modifications are not necessary on output devices with limited graphics capabilities.

This system currently supports four terminals, although the hardware is capable of supporting up to eight terminals. The addition of more simultaneous users would result in an increase in the system response delays. Our attempt was to minimize such delays because their effects in learning situations are not well known.

\section{IMPLEMENTATION-SOFTWARE}

The present version of ARIS was implemented to run in a UNIX ${ }^{1}$ environment and was written in the " $\mathrm{C}$ " programming language. The major objectives of the design and implementation of the software were flexibility, maintainability, and hardware independence. The efficiency of the resulting system was given secondary priority. The resulting system is highly modular and can easily be expanded and modified.

\section{General}

The ARIS system consists of two libraries, the executive module, a number of problem modules, and a few text and data files, as shown on Figure 2. In addition to these, there are several files used by the system for record keeping and progress evaluation, as well as index files for speeding up the access time to the text files

One of the nice features of ARIS is its reasonably good documentation, which consists of the two-volume ARIS manual. The first volume explains, in detail, the operation of the system and provides potential authors with the information required to generate new courseware. The second volume contains the details of the software implementation, including the source of all programs and subroutines. The initial instructions to students consist of a one-page text; all subsequent information is delivered on-line.

Executive module. At run time, the executive module takes control of the process and determines the next action to be taken based on the history of the student's interactions; that is, his performance and the last completed action. The global decisions made by the execu- 


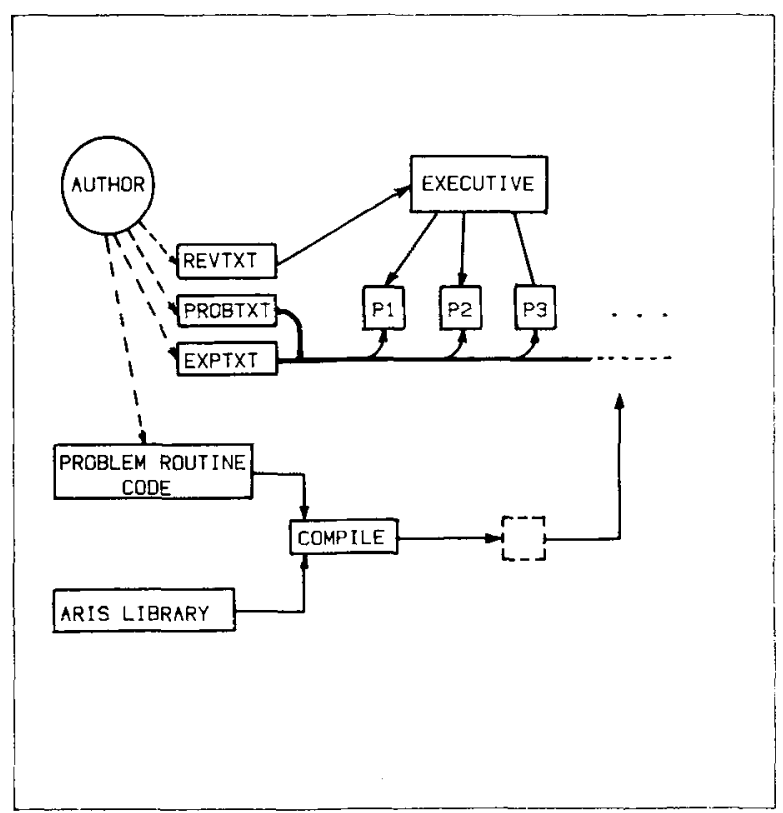

Figure 2. Overview of ARIS software. The executive and problem routines (P1, P2, P3, . . ) are executable programs, and the data base consists of three text files (revtxt, probtxt, and exptxt). An author developing new courseware appends the data base and, if necessary, develops a new problem routine.

tive program closely parallel the simplified flow diagram shown in Figure 1 and discussed above. In addition to the overall process control, the executive administers both the review of the material and the short quizzing. Then, depending on student's performance, the executive program selects the most appropriate homework problems. Following this selection, control is passed to one of a number of programs called problem routines that govern Phases 2 and 3 .

Problem routines. Each problem routine is a standalone program communicating with the executive module either by arguments passed along at execution time or by writing and reading the system text files. The problem routines are quite general, and each is capable of presenting a number of different specific problems. In addition, there are several variables in the problem routines whose values are determined at run time using a random number generator so that the same problem will require a different response each time.

The problem routines are executed during Phase 2 (homework assignment) and Phase 3 (homework testing). During the homework assignment phase, the problem routines simply assemble the problem statements and transmit them to the hard-copy output. During the homework testing phase, the problem routines control interactions with the student, which, depending on his responses, may be quite complex. An incorrect answer is handled in a way that enables the student to derive the maximum benefit from his errors; detailed explanations, together with quizzing, guide the student through the solution.
Text files. There are three text files containing the course material: revtxt, probtxt, and exptxt. The file revtxt contains the main text of the course, including the review text interspersed with short quizzes. The text in the file is divided into chapters, topics, paragraphs, and display frames. In addition to the text, revtxt contains commands that are interpreted at run time and mediate various digressions, such as questions, presentations to the student, or special display manipulations.

The probtxt file contains the text of the homework problems. The problem routines access this file to assign homework problems (Phase 2) and then again when the student is tested (Phase 3). The problems are organized by the topic tested and the type of the problem. In addition, the problems are classified by their difficulty. The last file, exptxt, contains text that is used optionally by the problem routines in the testing phase after an incorrect answer is received. The material follows the organization of the probtxt file and consists of in-depth explanations of problem solutions.

\section{Courseware Development Tools}

The most critical, time-consuming, and costly part of any computer-based instructional system is the development of high-quality courseware. Therefore, it is desirable that the authoring environment allows for efficient courseware development and that the resulting courseware be easily transportable to other systems. The ARIS authoring environment is limited to an extension of an already available general-purpose language. The decision to do this was based on the following considerations: (1) The principles for the design of optimal author languages are not well understood. (2) A highly modular design is desirable to enhance flexibility, expandability, and hardware and software independence. (3) Resource limitations did not allow for the basic research necessary to answer the critical design issues. The resulting software is embedded in the powerful " $\mathrm{C}$ " programming language and consists of a set of tools implemented as a library of subroutines and a number of general problem routines. Minimally, an author is required to master only a small subset of "C," but any additional knowledge allows him to fully appreciate and exploit the flexibility and power of the system.

The authoring tools included in the library enable the courseware developer to control the display, to monitor, interpret, and score student's responses, and to maintain detailed record keeping. The general problem routines are useful for a large number of different problems that have a similar structure. In addition, these routines can serve as models for the design of new problem routines.

\section{Proof Checker}

One of the especially useful and powerful features of ARIS is its ability to teach students to formally prove 


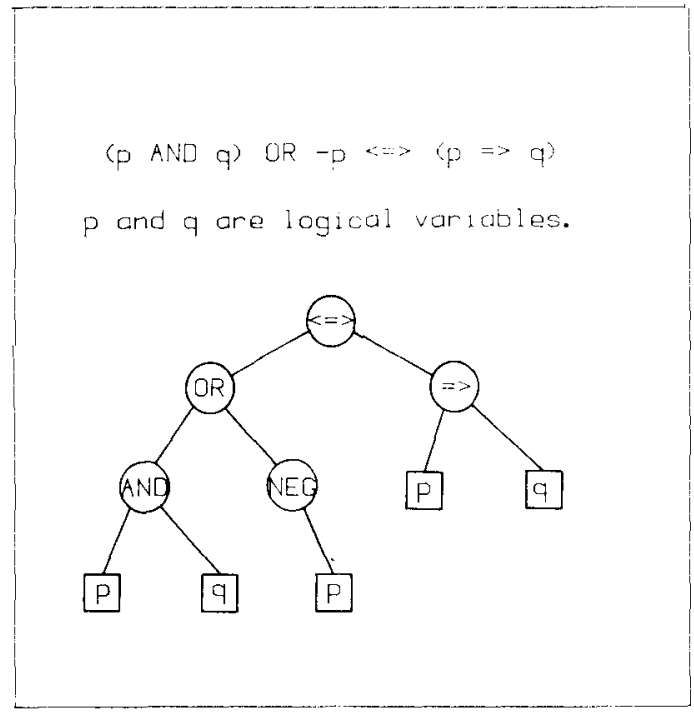

Figure 3. Example of a simple well formed formula and the corresponding parse tree. The nodes of the tree correspond to the logical connectives, and the leaves represent the sentential variables. The truth of the wff is evaluated by traversing and evaluating the tree for every possible assignment of values to the variables.

theorems. The same facility is used to mediate axiomatic proof checking and evaluation of translations from English to logic. The basic operation of the proofchecking programs involves parsing the student's input strings, constructing the corresponding parse tree, and then evaluating the implications of the parse tree.

Well formed expressions in logic belong to a class of context-free grammars and can therefore be described by a finite (and, in this case, small) set of production rules. The set of production rules, together with a few dominance and association rules, completely specifies the parsing operation. In ARIS, the parse trees are produced by a look-ahead left-to-right [LALR(1)] parser, constructed by a standard UNIX program called "YACC" (yet another compiler compiler). The output of YACC is a program that takes its input from a relatively primitive lexical analyzer that recognizes all logical connectives and literal variables and generates a binary tree. The internal nodes of the tree correspond to the logical connectives, and the terminal nodes represent variables. The root of the tree is the principal connective of the expression. A simple example illustrating the correspondence between a logical expression and its parse tree is shown in Figure 3.

Let us assume that a student entered the expression ( $p$ AND $q)$ OR $-p \Leftrightarrow(p \Rightarrow q)$, where $p$ and $q$ are logical variables. This expression can be represented by the parse tree shown in Figure 3.

Given a parse tree, the next step involves determining the correctness of the statement. A simple way to evaluate whether or not a logical expression is a tautology (always true) is by a recursive traversal and evaluation of this tree. The variables are assigned specific values, and each node of the tree representing a subexpression is evaluated for its truth value. If the root of the tree remains true for all possible "inputs," then the expression in question is a tautology.

\section{CONCLUSION}

Upon the completion of the initial development of ARIS, the system was used on a trial basis in a number of courses. These courses provide some data for a preliminary evaluation of the effectiveness and acceptabiltiy.

The system appeared to be particularly effective in allowing individuals to proceed at their own pace and thus to equalize differences in their backgrounds and their aptitudes.

To gauge the acceptability of the system to the students, we administered a questionnaire at the end of the course and asked them for reactions. The students' responses indicated that, in their opinion, the efficiency of learning and the precision of their knowledge after completing ARIS were superior to that of traditional classroom learning.

\section{NOTE}

1. UNIX is a trademark of Bell Laboratories. 